\title{
БОЛЕЗНЬ ИЦЕНКО-КУШИНГА. ТРУДНЫЕ ШАГИ К ДИАГНОЗУ
}

\author{
Шандалова И.В., Шимохина О.Н., Матвеева И.А., Руденко Т.Ю., Гржибовская О.Я. \\ ГБУз «Пензенская областная клиническая больница имени Н.Н. Бурденко», Пенза
}

ЦЕЛЬ: оценить возможности достижения ремиссии при своевременной диагностике, использовании новых методов лечения при болезни Иценко-Кушинга по данным регистра Пензенской области.

МАТЕРИАЛЫ И МЕТОДЫ: в РегИстре Пензенской области состоит 213 пациентов с нейроэндокринными опухолями гипоталамо-гипофизарной области. Из них с болезнью Иценко-Кушинга (кортикотропинома) - 16 человек (7,5\%). Средний возраст пациентов 40 лет (от 21 года до 64 лет); 2 мужчин (12,5\%), 14 женщин (87,5\%). К диагнозу больные шли от 1 года до 4 лет, наблюдаясь у травматологов - ортопедов, ревматологов кардиологов, неврологов,окулистов.

РЕЗУЛЬТАТЫ: на момент постановки диагноза:

1) характерная внешность (перераспределение подкожно-жирового слоя по кушингоидному типу, матронизм, стрии) - 14 чел. (87,5\%);

2) стероидный сахарный диабет - 11 человек (68,8\%), нарушенная толерантность к углеводам 5 человек (31,3\%);

3) стероидная кардиомиопатия - 6 человек $(37,5 \%)$;

4) артериальная гипертензия - 15 человек (93,8\%);

5) нарушение менструального цикла - 8 человек (50\%);

6) остеопороз - 7 человек (43,8\%);

7) остеопения - 2 человека (12,5\%); боли в костях - 8 человек (50\%); боли в суставах - 8 человек (50\%);

8) мышечная слабость - 9 человек (56,3\%); общая слабость - 13 человек (81,3\%).

У 16 пациентов обнаружена аденома гипофиза (кортикотропинома): микроаденома - 12 человек (75\%), макроаденома - 4 человека (25\%); эндоселлярный рост аденомы - 2 человек (50\%), параселлярный рост - 1 человек (25\%), супраселлярный рост - 1 человек (25\%). Клинико-лабораторные методы, подтверждающие гиперкортицизм - 16 человек (100\%)

Все пациенты были направлены в ФГБУ «НМИЦ эндокринологии» г. Москвы. Лечение: эндоскопическое трансназальное удаление аденомы произведено 13 пациентам (81,3\%), повторное эндоскопическое трансназальное удаление аденомы - 4 пациентам (30,8\%), 1 пациенту (6,25\%) проведена двусторонняя адреналэктомия, 3 пациентам (18,75\%) - протонотерапия, 2 пациентам (12,5\%) — гамма-нож; у одной пациентки (6,25\%) произошло кровоизлияние в аденому гипофиза, развилась вторичная надпочечниковая недостаточность, требующая проведения заместительной терапии. Стадии ремиссии достигли 11 человек $(68,8 \%)$, неполной ремиссии - 2 человека (12,5\%). Отсутствует ремиссия у 3 человек (18,8\%), одному пациенту после двукратного эндоскопического трансназального удаления аденомы гипофиза и лучевой терапии (гамма-нож), при отсутствии ремиссия заболевания, назначено медикаментозное лечение препаратом Пасиреотид.

ВЫВОдЫ: сложность и многообразие клинических проявлений кортикотропиномы —опухоли гипоталамо-гипофизарной области ведет порой к несвоевременной постановке диагноза, развитию тяжелых осложнений, в связи с чем необходимо ориентировать прежде всего врачей первичного звена здравоохранения на выявление данной патологии на ранней стадии заболевания. Раннее выявление и адекватное лечение нейроэндокринных опухолей гипоталамо-гипофизарной области позволяют добиться хороших результатов.

КЛЮЧЕВЫЕ СЛОВА: болезнь Иченко-Кушинга; регистр; аденома гипофиза; ремиссия. 